This document is confidential and is proprietary to the American Chemical Society and its authors. Do not copy or disclose without written permission. If you have received this item in error, notify the sender and delete all copies.

\title{
Dynamical Interconversion between Excitons and Geminate Charge Pairs in Two-Dimensional Perovskite Layers Described by Onsager-Braun Model
}

\begin{tabular}{|r|l|}
\hline Journal: & The Journal of Physical Chemistry Letters \\
\hline Manuscript ID & jz-2019-03709b \\
\hline Manuscript Type: & Letter \\
\hline Author: & 15-Dec-2019 \\
\hline Complete List of Authors: & $\begin{array}{l}\text { Mondal, Navendu; University of Texas at Dallas, Physics } \\
\text { Naphade, Rounak; King Abdullah University of Science and Technology, } \\
\text { physical science and Engineering Division } \\
\text { Zhou, Xiaohe; University of Texas at Dallas, Physics } \\
\text { Zheng, Yangzi; University of Texas at Dallas, Physics } \\
\text { Lee, Kwangjae; King Abdullah University of Science and Technology, } \\
\text { Physical Science and Engineering Division } \\
\text { Gereige, Issam; Saudi Aramco Research \& Development Center, } \\
\text { Al-Saggaf, Ahmed; Saudi Aramco Research \& Development Center } \\
\text { Bakr, Osman; King Abdullah University of Science and Technology, } \\
\text { Division of Physical Sciences and Engineering, Solar and Photovoltaics } \\
\text { Engineering Center; King Abdullah University of Science and Technology, } \\
\text { Division of Physical Science } \\
\text { Mohammed, Omar; King Abdullah University of Science and Technology, } \\
\text { Chemical and Materials Science Programs } \\
\text { Gartstein, Yuri; University of Texas at Dallas, Department of Physics } \\
\text { Malko, Anton; University of Texas at Dallas, Physics }\end{array}$ \\
\hline
\end{tabular}

\section{SCHOLARONE ${ }^{\text {IM }}$ Manuscripts}




\title{
Dynamical Interconversion between Excitons and Geminate
} Charge Pairs in Two-Dimensional Perovskite Layers Described by

\section{Onsager-Braun Model}

Navendu Mondal', Rounak Naphade, ${ }^{2}$ Xiaohe Zhou', Yangzi Zheng ${ }^{1}$, Kwangjae Lee ${ }^{2}$, Issam Gereige $^{3}$, Ahmed Al-Saggaf ${ }^{3}$, Osman M. Bakr ${ }^{2}$, Omar F. Mohammed ${ }^{2}$, Yuri N. Gartstein ${ }^{1}$, and Anton V. Malko ${ }^{1 *}$

${ }^{1}$ Department of Physics, The University of Texas at Dallas, Richardson, Texas 75080, United States

${ }^{2}$ Division of Physical Sciences and Engineering, King Abdullah University of Science and Technology, Thuwal 23955-6900, Kingdom of Saudi Arabia

${ }^{3}$ Saudi Aramco Research \& Development Center, Dhahran 31311, Kingdom of Saudi Arabia

\begin{abstract}
KEYWORDS. 2D hybrid perovskite, PL spectroscopy, femtosecond transient absorption, excitons, geminate charge pairs, kinetic-diffusion model.
\end{abstract}




\begin{abstract}
Time-resolved photoluminescence (PL) and femtosecond transient absorption (TA) spectroscopy are employed to study the photoexcitation dynamics in a highly-emissive two-dimensional perovskite compound (en $)_{4} \mathrm{~Pb}_{2} \mathrm{Br}_{9} .3 \mathrm{Br}$ with the ethylene diammonium (en) spacer. We find that, while the PL kinetics is substantially $T$-dependent over the whole range of studied temperatures $T$ $\sim 77-350 \mathrm{~K}$, the PL quantum yield remains remarkably nearly $T$-independent up to $T \sim 280-290$ K appreciably decreasing only at higher temperatures. Considerable differences are also revealed between the TA spectra and responses to the excitation power at low and at room temperatures. Numerical solutions of Onsager-Braun-type kinetic-diffusion equations illustrate that the salient features of the experimental observations are consistent with the picture of a $T$-dependent dynamic interplay between tightly bound emissive excitons and larger-size, loosely bound, non-emissive geminate charge pairs arising already at earlier relaxation times. The geminate pairs play the role of "reservoir" states providing a delayed feeding into the emitting excitons thus giving rise to the longer-time PL decay components and accounting for a stable PL output at lower temperatures. At higher temperatures, the propensity for thermal dissociation of excitons and bound pairs increases subsequently leading to the precipitous decrease of the PL.
\end{abstract}


Perovskites materials of different dimensionalities have recently attracted considerable attention due to both fundamental interest and prospective photonic applications such as for lasers, ${ }^{1,2,3}$ light-emitting diodes (LEDs), ${ }^{4,5,6}$ photodetectors ${ }^{7}$ and X-ray scintillators. ${ }^{8}$ In particular, two-dimensional (2D) layered perovskites exhibit greater long-term stability, tunability and increased exciton binding energies $\left(E_{b}^{e x c} \gtrsim 0.1 \mathrm{eV}\right.$ ) compared to the three-dimensional counterparts owing to the quantum and dielectric confinement similar to $2 \mathrm{D}$ atomic layers and semiconductor quantum wells. ${ }^{9,10,11}$ In the ubiquitous case of Ruddlesden-Popper perovskites (RPPs) with a general composition $R_{2} A_{n-1} B_{n} X_{3 n+1}$, a group of $n$ layers of connected inorganic octahedra ( $A$-central cation, $B$-metal, $X$-halide) is separated from other groups by larger organic cations $R$. It should be noted that the bandgap $E_{g}$ of these systems is stoichiometrically affected by the R/A ratio. ${ }^{9}$

Optimizing the performance of hybrid perovskite devices calls for a detailed understanding of their photophysical properties depending on nuances of exciton and charge carrier generation and recombination, photoluminescence (PL) as well as nonradiative losses. While sample-dependent monomolecular non-radiative recombination may be prevalent at low excitation densities, ${ }^{12,13}$ exciton-exciton annihilation can limit PL efficiency at higher excitation fluences. ${ }^{14}$ The excitation dynamics in mixed-phase RPPs was also reported to be strongly affected by charge or/and energy transfer processes..$^{15,16,17}$ As the confinement is known to enhance the role of interactions in lowerdimensional systems, in addition to the increased excitonic (electron-hole Coulomb attraction) effects, one may expect the increased effects of the electron interaction with the surroundings (other degrees of freedom such as the underlying lattice distortions). Polaronic effects have been invoked for both excitons (self-trapped excitons ${ }^{18,19}$ ) and charge carriers (electron- and holepolarons) in the photophysics of hybrid perovskites. ${ }^{20,21,22,23}$ Because of these complexities, 
interpretations of the experimentally measured PL kinetics provide complicated and, at times, conflicting pictures.

Here we explore the photoexcitation dynamics in a highly emissive $n=12 \mathrm{D}$ perovskite (en) ${ }_{4} \mathrm{~Pb}_{2} \mathrm{Br}_{9} .3 \mathrm{Br}$ (thereafter termed as MAen20) with the ethylene diammonium (en) as spacer synthesized with the modified protocol. We employed time-resolved PL and femtosecond transient absorption (TA) spectroscopy over a wide temperature range $(T \sim 77-350 \mathrm{~K})$. While the initial amplitude, $f(t=0)$, of the time-resolved PL kinetics exhibits a monotonous decrease with the increase of temperature, the PL decay also becomes progressively slower in the $T \sim 77-280 \mathrm{~K}$ range so that both PL spectra and integrated PL kinetics $\int f(t) d t$ remarkably remain nearly $T$ independent in this range. The time-dependent character of the PL decay curves, $f(t)$ changes from nearly monoexponential behavior $(\tau \sim 2.2-2.5 \mathrm{~ns})$ at lowest temperatures to the appearance of other clearly resolvable, slower components at elevated temperatures. Only at $T$ above $\sim 280 \mathrm{~K}$, the PL intensity exhibits a precipitous drop accompanied by acceleration of the PL decay. Pumpprobe measurements also reveal substantial differences in the TA spectra at low and high temperatures with the disappearance of the exciton-exciton annihilation effect at cryogenic temperatures. We employed numerical solutions of the combined Onsager-Braun-type $24,25,26$ kinetic-diffusion equations to model the time evolution of the photoexcitation ensemble. The observed behavior is consistent with the picture of $T$-dependent dynamic interplay between tightly bound emissive excitons and larger-size, loosely bound, non-emissive geminate charge pairs that act as "reservoir" states, providing a feedback mechanism into the emitting excitons and giving rise to the longer decay components. Overall, our observations provide a unified picture of the excitation dynamics in 2D perovskites where the thermal dissociation of excitons and bound pairs leads to the precipitous decrease of the PL at high temperatures. 
The schematics of single crystal synthesis is shown in Figure 1. The single crystals of MAen20 perovskite sample were prepared via a modified acid cooling protocol in the form of narrow microflakes (average length $\sim 100-150 \mu \mathrm{m}$, width and thickness $15-20 \mu \mathrm{m}$ ) that allowed visualization of the individual entities and minimized light's reabsorption. ${ }^{27}$ Figure $1(\mathbf{b}, \mathbf{c})$ shows the arrangement of $[\mathrm{Pb}-\mathrm{Br}]$ octahedrons which form alternate ladder-like structure as discussed in the earlier report. ${ }^{28}$ To confirm the phase purity of our crystals, we performed powder X-ray diffraction (PXRD) of the bulk crystals and compared it with the simulated PXRD pattern, Figure 1(d). The PXRD pattern matches well with the simulated pattern and confirms the phase purity of the final crystals. We also measured nuclear magnetic resonance ( ${ }^{1} \mathrm{H}$ NMR and $\left.{ }^{13} \mathrm{C} N M R\right)$ spectra in liquid state by dissolving the as formed crystals in the $\mathrm{d}_{6}$ DMSO solution. The NMR results are provided in the supporting information, Figure S1. The ${ }^{1} \mathrm{H}$ NMR result clearly shows the presence

(a)
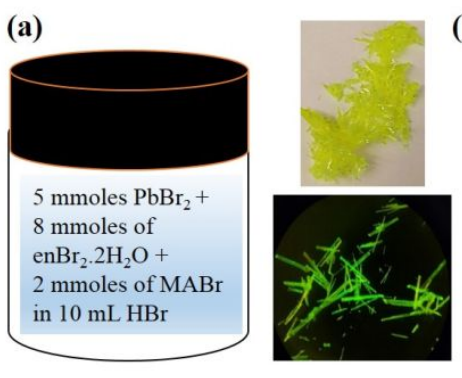

(b) ${ }^{b} ?^{\mathrm{a}}$

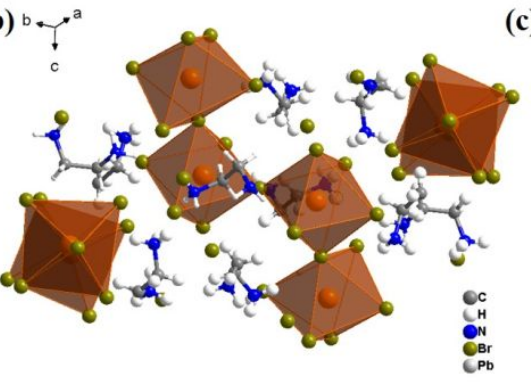

(c)
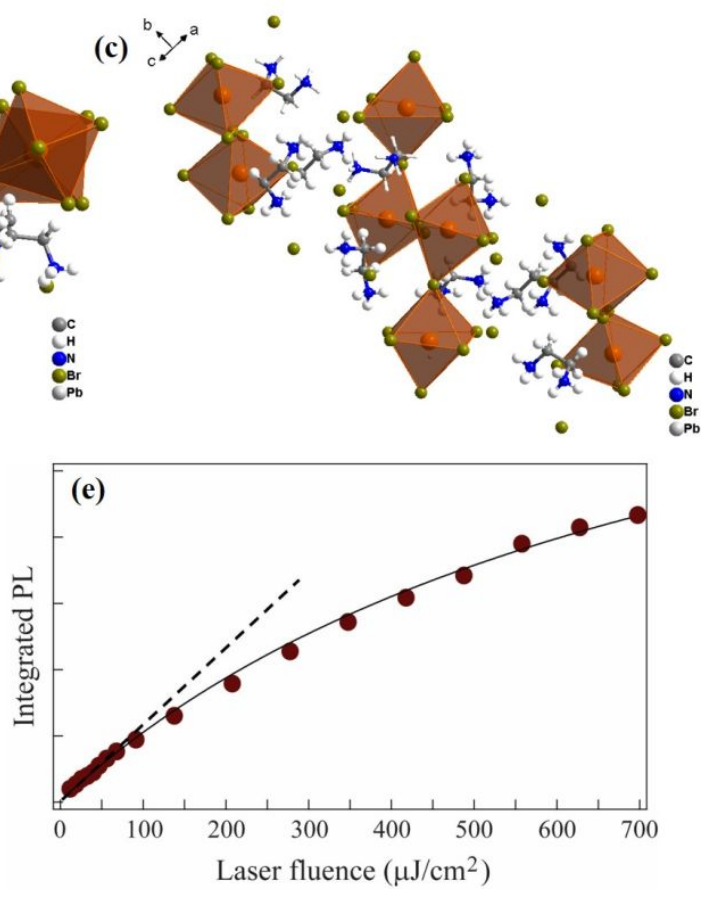

Figure 1. (a) Schematic of single crystal synthesis by acid cooling method and the photographs of microflakes obtained under ambient light and $405 \mathrm{~nm}$ blue light irradiation. (b, c) structure of 2D MAen20 perovskite. (d) PXRD pattern for the 2D crystals with simulated XRD pattern obtained from the single crystal XRD analysis. (e) PL fluence dependence at $400 \mathrm{~nm}$ excitation. Solid line - fit to the saturation curve, dotted line - linear regime. 
of only one organic cation (i.e. $\left.\mathrm{En}^{2+}\right)$. We did the quantification of methylene $\left[\left(-\mathrm{CH}_{2}-\mathrm{CH}_{2-}\right) \delta=\right.$ $3.02 \mathrm{ppm}]$ and ammonium $\left[\left(-\mathrm{NH}_{3}\right) \delta=7.77 \mathrm{ppm}\right]$ protons by integration and the ratio of $2: 3$ matches well with the enBr 2 salt composition, i.e. $\mathrm{Br}-\mathrm{NH}_{3}-\mathrm{CH}_{2}-\mathrm{CH}_{2}-\mathrm{NH}_{3}-\mathrm{Br}$. More details of the synthesis procedure and description of time-resolved detection techniques are presented in the Supporting Information (SI). Measured PL quantum yield (PLQY) in the bulk form was 30\%, however, our previous experience with $\mathrm{PbBr}$-based microwires have shown considerably larger PLQY in an individual wire.27 Figure 1(e) shows room temperature PL emission as a function of the excitation laser fluence, $F$ and indicate the geminate character of the exciton recombination (non-geminate recombination would scale quadratically with $F$ at low fluences). We found linear excitation regime up to $F \sim 100 \mu \mathrm{J} / \mathrm{cm}^{2}$ level, followed by saturation behavior due to the appearance of the non-radiative recombination processes. Observed excitonic behavior and saturation parameters correspond well with recently published work concerning PL properties the methylammonium-based RPP layers ${ }^{14}$ and in line with expectations for this type of material as was recently discussed in the context of the dielectric properties of the organic cations.11

To assess the dynamics of recombination in RPPs, we turned our attention to the PL kinetics, measured across a range of excitation fluences and temperatures. Within the linear range of excitation fluences the observed PL dynamics did not change appreciably, while at much higher excitation fluencies $\left(F>300 \mu \mathrm{J} / \mathrm{cm}^{2}\right)$, we indeed observed acceleration of the early part of the PL dynamics, Figure S2, indicating an onset of higher-order non-radiative recombination processes such as exciton-exciton annihilation. Figure 2(a) illustrates representative PL dynamics in the linear regime taken at $F=70 \mu \mathrm{J} / \mathrm{cm}^{2}$ fluence in the temperature range $80-335 \mathrm{~K}$ (full range of kinetics is shown in Figure S3). Recombination dynamics shows nearly perfect monoexponential decays from $\mathrm{T}=77 \mathrm{~K}$ to about $\mathrm{T} \sim 150 \mathrm{~K}$ with time constant $\tau_{1} \sim 2.2-2.5 \mathrm{~ns}$. This value corresponds 
well to characteristic lifetimes reported in a number of publications concerning layered perovskites that include chemically and epitaxially grown 2D RPPs ${ }^{29,30}$ as well as exfoliated ones. ${ }^{31}$ Hence, we ascribe this lifetime to the exciton recombination. Upon the increase of $T$, however, the PL decay curves start to exhibit continuous evolution and now are better described by two time constants. In addition to $\tau_{1}$, the second time constant, $\tau_{2} \sim 5-7$ ns (inset in Figure 2(a), long component) is observed and closer to the room temperature (RT), the decays become decidedly multiexponential. The parameters of a numerically accurate multi-exponential representation of the normalized PL dynamics, $f(t) / f(0)=\sum_{i} A_{i} \exp \left(-t / \tau_{i}\right)$, at different temperatures are tabulated in Table S1. As the inset in Figure 2(a) also illustrates, the initial PL decay rate $\sum_{i}\left(A_{i} / \tau_{i}\right)$ gradually decreases with growing $T$ up to about $280-290 \mathrm{~K}$, and starts increasing afterwards. The initial PL amplitude $f(0)$, on the other hand, consistently decreases over the whole range of temperatures. In contrast, the time-integrated PL kinetics: $\int f(t) d t=f(0) \sum_{i} A_{i} \tau_{i}$, stays nearly $T$-independent up to about $280 \mathrm{~K}$. Figure 2(b) shows that the temperature variation of the evaluated integrated PL kinetics is in good correspondence with the temperature variation of the independently observed peak intensities in the PL spectra. The fact that the latter along with the spectrally integrated PL exhibit nearly constant values across the 77-290 K temperature range implies that overall PLQY remains practically unchanged in this range.

A multiexponential character of the PL decay in the linear fluence regime has been frequently ascribed to the existence of different population of excitons (emissive species). ${ }^{32,33} \mathrm{We}$, however, observe that the initial amplitudes $f(0)$ of PL decays, that is, the number of the initially available emissive species consistently decreases with the growing temperature. The dynamics that we measured suggests the coexistence of emissive and non-emissive species that can convert into each 

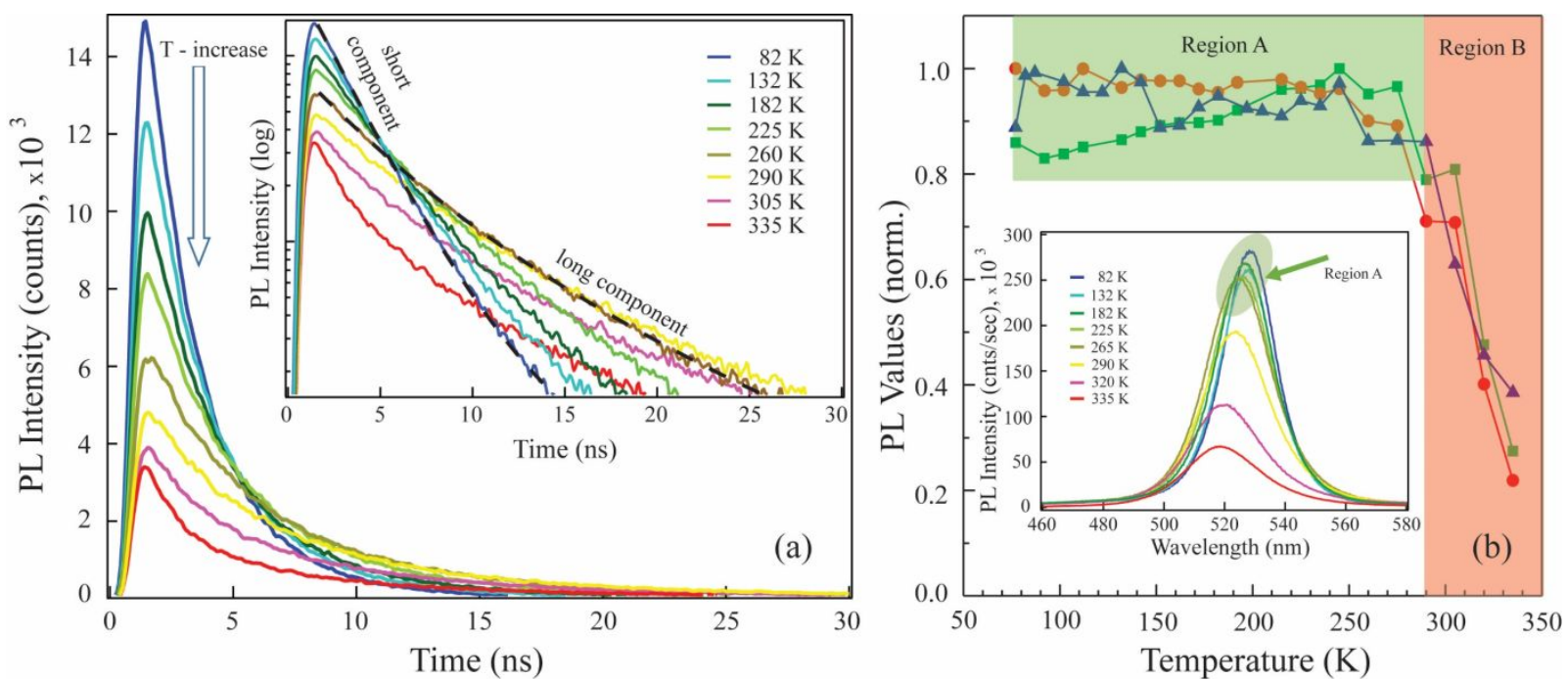

Figure 2. (a) $\mathrm{PL}$ dynamics at $\mathrm{F}=70 \mu \mathrm{J} / \mathrm{cm}^{2}$ taken in the temperature range $77-335 \mathrm{~K}$ recorded at the maxima of $\mathrm{PL}$ spectra. Inset: Same traces plotted on the logarithmic scale. Dotted black lines - bi-exponential fits. (b) Peak values of PL spectra (red circles), integrated values of PL spectra (green squares) and integrated PL dynamics (blue triangles) as a function of temperature. All values normalized to the maximum. Inset: Representative PL spectra used to extract PL values.

other. Note that the observed higher- $T$ decrease in the PL intensity is in fact common in perovskite materials. When interpreted as due to the excitons' thermal dissociation into free electrons and holes, this decrease would frequently be used for assessing the exciton binding. ${ }^{10,13}$ One should also recognize that spatially separated geminate charge pairs (e.g., in the form of electron- and hole-polarons as a consequence of the strong interaction with the environment) can be present at lower temperatures as well, where they are still well bound by the mutual Coulomb attraction. Such bound (more loosely and larger radius) charge pairs could play a role of non-emissive excitation species while the tightly bound and smaller-radius electron-hole pair in the excitonic state would represent an emissive species. The dynamic balance (inter-conversion) between the loosely bound charge pairs and excitons is $T$-dependent: lower the temperature, larger is the relative proportion of the excitons resulting in a faster decay of the whole family of excitations while maintaining the overall PLQY. Only at sufficiently high temperatures, a substantial number 
of excitons will be efficiently dissociating out of the Coulomb capture region to form the loosely bound charge pairs, leading to the decrease of the excitonic emission.

The framework of the geminate charge pairs that can undergo both recombination and spatial separation is well known in the photophysics of organic and amorphous solids and was studied in different modifications since the original work of Onsager (see reviews in Refs. 25-26 for numerous original references). Here we explore an application of an Onsager-Braun-type model by numerically solving combined kinetic-diffusion equations for the time evolution of the exciton and charge-pairs ensemble. As shown schematically in Figure 3(a), the excitons are characterized by time-dependent number $n(t)$ while the geminate charge pairs by the $2 \mathrm{D}$ pair distribution function $\rho(r, t)$ defined for pair separation distances $r \geq a$ so that number of pairs $n_{p}(t)=$ $\int_{a}^{\infty} 2 \pi r \rho(r, t) d r$. The time-dependent PL intensity is proportional to $n(t)$, whose variation in time:

$$
\frac{d n}{d t}=-\gamma n-I(t), \quad I(t)=2 \pi a j(a, t)=\alpha n(t)-\kappa \rho(a, t), \quad \text { (1) exhibits decay with }
$$

rate $\gamma$ (may include both radiative and non-radiative recombination) but is modified by current $I$ ( $t$ ) due to the exchange with the pairs manifold. Here coefficient $\alpha$ quantifies the conversion of excitons into pairs while coefficient $\kappa$ the reverse conversion of pairs into excitons at the "boundary", $r=a$. At the same time, this current $I(t)$ represents the boundary condition for the 2D radial current density $j(r, t)$ in the spatiotemporal evolution of the pair distribution function:

$$
\frac{\partial \rho}{\partial t}=-\frac{1 \partial}{r \partial r}(r j)-\gamma_{1} \rho, \quad j=-D\left(\frac{\partial \rho}{\partial r}-\frac{F}{k_{B} T} \rho\right)
$$




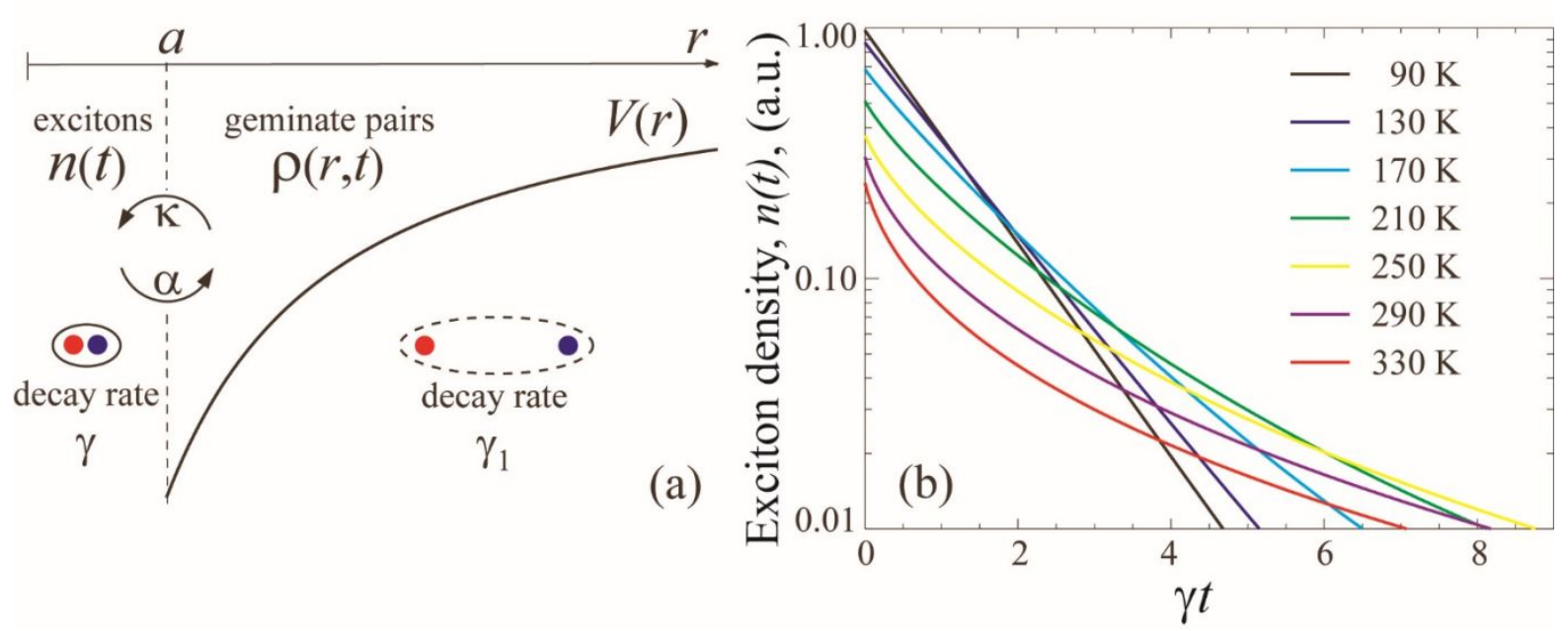

Figure 3. (a) Model schematics of the interconversion between excitons (emissive species) and geminate pairs (nonemissive species). (b) An example of the temporal evolution of the number of the emissive species for different temperatures simulated with a set of model parameters, see the text.

where the $2 \mathrm{D}$ continuity equation is supplemented by the phenomenological pair decay with rate $\gamma_{1}$ due to, say, trapping and other non-radiative channels. The current density in Eq. (2) is composed of the diffusion and drift components ( $D$ is the diffusion coefficient and Einstein's relationship utilized for the mobility), where force $F=-\partial V / \partial r$ is determined by the effective interaction potential energy $V(r)$ between charges in the geminate pair. Traditionally, $V(r)$ used is just the Coulomb attraction, leading to $F / k_{B} T=-r_{C} / r^{2}$ with the Coulomb capture radius $r_{C}=$ $q^{2} / 4 \pi \varepsilon_{0} \varepsilon k_{B} T$. It should be clear, however, that the effective interaction could be modified due to e.g. polaronic effects. A reduction of $r_{C}$ with growing temperature $T$ would manifest in the increasing propensity for thermal dissociation of the pairs. A similar strong effect of the rising $T$ on the increasing relative number of geminate pairs $n_{p}$ with respect to the number of excitons $n$ would follow from the $T$-dependent model coefficient ratio $\alpha / \kappa \propto \exp \left(-U / k_{B} T\right)$, where $U$ is an effective activation energy for the process of interconversion between excitons and geminate 
pairs. The diffusion coefficient $D$ can also depend on $T$, consistently with the operating scattering processes.

Given the initial conditions $n(0)$ and $\rho(r, 0)$, the framework of coupled equations (1) and (2) allows one to find the time dependence of $n(t)$ that can be compared to the experimentally observed time-resolved PL kinetics $f(t)$. These initial conditions (distributions) are determined by the relaxation processes following the original higher-energy laser photoexcitation of the system, which are frequently subsumed under the label of "thermalization". We do not know a priori what this initial distribution should be in 2D perovskites. Evidently, some of the limiting pictures would be inconsistent with our observations. For instance, in a purely ballistic picture, the initial condition could be all geminate pairs at some thermalization distances $r$. Then the signal $f(t)$ from excitons would feature PL kinetics risetimes, which we do not observe at all in the experiment. In the opposite limit of complete "internal conversion", the initial condition could be all excitons. Then the PL would be starting from the same $f(0)$ values at different temperatures, also not recorded in the PL measurements. Instead, our observations suggest that the relaxation results in "balanced" $T$-dependent initial distributions with the relative number of emissive species (proportional to $f(0)$ ) decreasing with the rising $T$. For our illustrative purposes here, we chose therefore to try initial distributions generated by running ("relaxing") our model at different temperatures from the purely excitonic states for some time. The resulting patterns of $n$ and $\rho(r)$ were then renormalized to the common initial condition $n(0)+n_{p}(0)=1$, from which the model runs were resumed. Figure 3(b) exemplifies results for $n(t)$ that were obtained this way with a set of model parameters (in particular, the overall exciton thermal ionization energy in this example corresponds to $1350 \mathrm{~K}$ and $\left.\gamma_{1}=0.05 \gamma\right)$. When compared to PL data in Figure 2(a), the model kinetics clearly reproduces all important experimental observations - slowing down of the initial decay with growing $T$ for 
lower temperatures followed by an acceleration of that decay at higher temperatures. At the same time, the progression of slower decay components at elevated temperatures comes out as a natural consequence of the dynamic interplay between excitons and the bound pairs manifold. Besides improvements in specifications of initial conditions, qualitative model refinements need to be further explored, including disorder effects that may lead to distributions (rather than some specific values) of the model parameters.

As the PL data only reflects the evolution of the emissive states at $>100$ ps time resolution, we also performed a femtosecond TA study that probes faster responses in a wide spectral range, including from non-emissive species. ${ }^{34}$ Our femtosecond TA experiment allows us to study absorption in the individual microflakes, thus directly comparing the results to PL data. Figure 4 compares the time-dependent evolution of the TA spectra and fluence-dependent bleach dynamics at low $(77 \mathrm{~K})$ and at room $(\mathrm{RT})$ temperatures. While the TA spectra are characterized by the pronounced bleach signals at 520-530 nm (associated with the excitons responsible for the PL signal, Figure S4) at both temperatures, they also exhibit a number of pronounced differences. In particular, the RT spectra, Figure 4(a), exhibit a long-lived ( $>3.5 \mathrm{ns,} \mathrm{longer} \mathrm{than} \mathrm{our} \mathrm{delay} \mathrm{range,}$ Figure S5) photoinduced absorption (PA) component, extending from $550 \mathrm{~nm}$ to beyond $700 \mathrm{~nm}$ (our detection limit). Its presence and lifetime correlate well with the existence of longer PL components in the RT PL dynamics. We note that some previous publications related such longlived broad PA to the production of free electrons and holes, characterized by Drude-like conductivity. ${ }^{35}$ Our PA signals exhibit linear fluence dependence shown in the inset in Figure 4(a). Figure 4 (b) shows the RT bleach dynamics normalized at the long times for different excitation levels. At the excitation densities above linear regime $\left(F>100 \mu \mathrm{J} / \mathrm{cm}^{2}\right)$ we observe emergence of the fast, sub-100 ps components, indicative of the non-linear annihilation processes. 

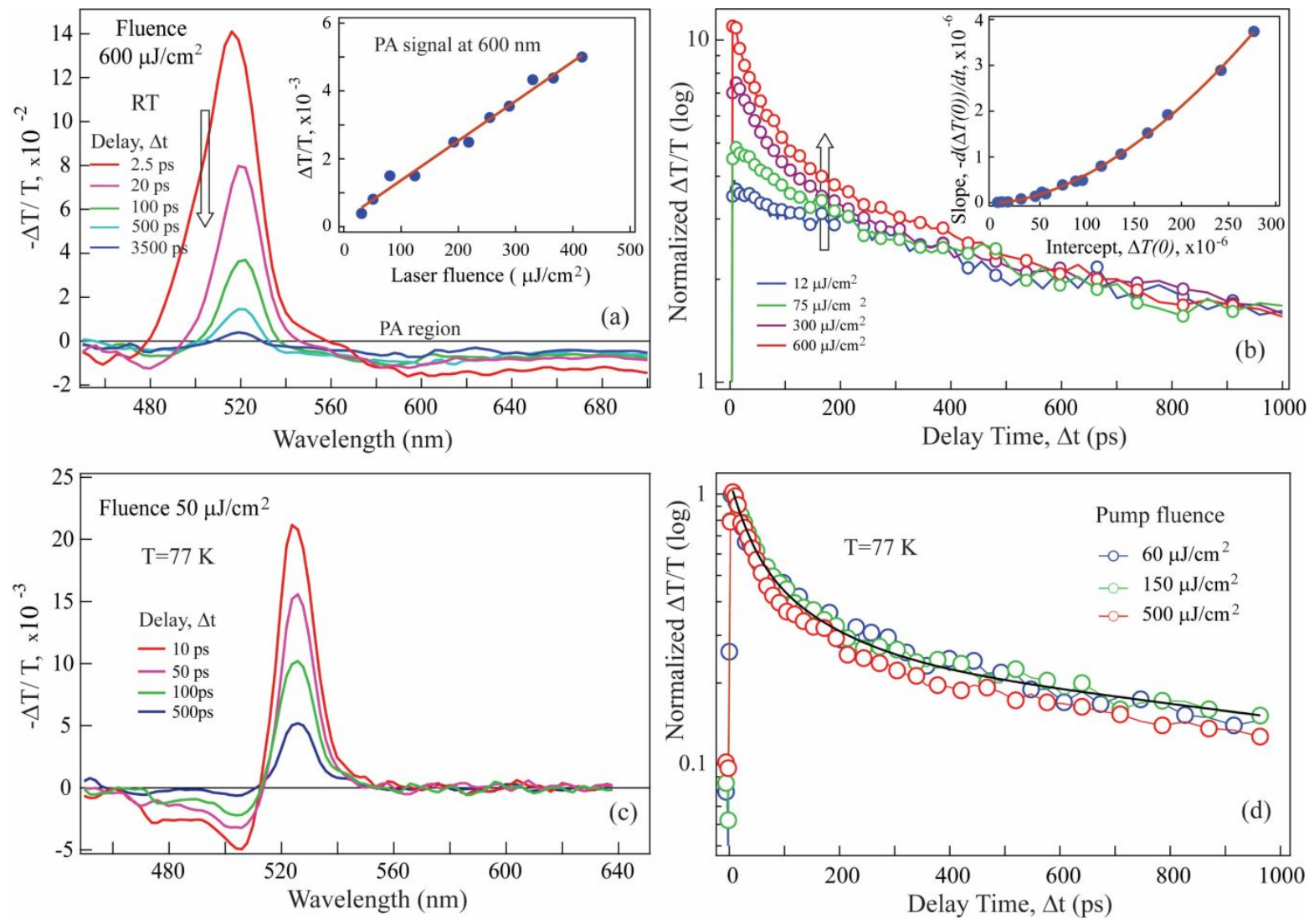

Figure 4. (a) Time evolution of the TA spectra of (MAen20) 2D perovskites at RT and high fluence. Inset: excitation fluence dependence of the PA region at $600 \mathrm{~nm}$. (b) RT bleach dynamics at $520 \mathrm{~nm}$ at different fluences, with dynamics normalized to match at long times. Inset: Slope of the early time curves vs. initial amplitude. (c) Time evolution of TA spectra at $77 \mathrm{~K}$ in the linear fluence regime. (d) Bleach dynamics at different fluences at $77 \mathrm{~K}$.

If one were to employ the generic kinetic equation $d n_{e h} / d t=-k_{1} n_{e h}-k_{2} n_{e h}^{2}$ for the photoexcitation $n_{e h}$ dynamics, the non-linear (bimolecular type) annihilation would be described by the second term with kinetic coefficient $k_{2}$. Owing to the very different recombination timescales (radiative recombination on the $n s$ scale and pair annihilation at less than $100 \mathrm{ps)} \mathrm{we} \mathrm{can}$ separate early time dynamics. The inset in Figure 4(b) shows the slope of the population dynamics at early times (i.e. $\propto-d(\Delta T) / d t$ at $t=0$ ) as a function of the initial photoexcitation density $n_{e h}$ $(0) \propto \Delta T(0)$. Full set of un-normalized dynamics is available in Figure S6. It nicely follows the quadratic behavior at higher densities, proportional to the non-linear $k_{2}$ term. ${ }^{36}$ In contrast, Figure 
4(c) shows TA spectra at $77 \mathrm{~K}$ that do not possess broadband PA manifold and no discernible fluence dependence is observed in the TA spectra and bleach dynamics at this low $T$. Bleach dynamics, shown in Figure 4(d), illustrate instead a common behavior characterized by a twoexponential decay with $\tau_{\text {fast }} \sim 50-80$ ps and $\tau_{\text {slow }}>1$ ns (limited by the delay range). The slower component clearly corresponds to lifetime $\tau_{1}$ observed in the PL emission. The fast component indicates a non-radiative channel with a monomolecular type recombination (as would be described by kinetic $k_{1}$ coefficient above), most likely related to surface/ligand-induced trapping as is long known for colloidal nanocrystals ${ }^{37}$ and was recently shown in 2D RPP compounds as well. ${ }^{38}$ Being on a sub-100 ps timescale, these are not resolvable in PL dynamics, however, and its time-integrated contribution is small, less than $30 \%$ of the total decay and in-line with high PLQY of our samples.

The observed differences in the TA responses at $77 \mathrm{~K}$ and at RT would be consistent with our picture of the $T$-dependent composition of the photoexcitation population developing already at earlier relaxation times: while being predominantly consistent of excitons at $77 \mathrm{~K}$, it would feature a substantial number of charge pairs at RT. Indeed, then the long-lived broad PA absorption associated with individual charges would be observed at RT but not at $77 \mathrm{~K}$. At the same time, the difference in the fluence dependence would be related to the difference in the physical sizes of excitons and charge pairs. Based on the linear absorption data from individual microflakes in our samples and assuming a close-packed arrangement of the octahedral layers, a rough estimate of the initial photoexcitation density at $F=300 \mu \mathrm{J} / \mathrm{cm}^{2}$ fluence yields $n_{e h}(0) \sim 10^{11} \mathrm{~cm}^{-2}$ in a single layer. This density corresponds to the average in-plane distance between the excitations $l=$ $1 / \sqrt{\pi n_{e h}(0)} \sim 20 \mathrm{~nm}$, which would be much larger than the typical exciton Bohr radius in many 2D perovskites. ${ }^{39}$ However, being the larger-size entities, the charge pairs may experience 
substantial spatial overlap and start to non-radiatively annihilate each other at much lower fluences as compared to smaller-size emissive excitons. Additionally, exciton diffusion is likely to be slower at $77 \mathrm{~K}$, further preventing exciton-exciton annihilation.

The Onsager framework of the geminate charge pair dynamics assumes a sufficiently strong interaction of electrons with the environment. An extensive literature on hybrid perovskites in fact discusses the importance of the effects of the static and dynamic disorder as well as the existence of polarons. ${ }^{22,23}$ It is known that self-localization of electrons into polaronic states can generally be driven by both short- and long-range interactions with the underlying lattice, and the long-range polarization is omnipresent in perovskites due to the polar nature of their structure. ${ }^{40}$ It is therefore possible that charges in the geminate pair could be in the form of electron- and hole-polarons. The analysis of our experimental data, however, does not rely on a specific microscopics of charge carriers. While our experiments also do not address directly the nature of the emissive excitonic states, a clear pattern is exhibited of the PL emission spectra both in terms of the spectral composition and lifetimes. It has been discussed that excitons in 2D RPPs can undergo selftrapping (STEs) via exciton-phonon coupling driven lattice distortions. ${ }^{41}$ The primary experimental identification of STEs is related to the appearance of broadband "white light" emission below the optical gap, ${ }^{42}$ with PL signatures that are further enhanced at low temperatures. Additionally, formation and emission decay of STEs follow the lattice relaxation dynamics, typically on a ps time scale. In our observations, however, the presence of a long-lived TA signal is not accompanied by distinct low-energy PL bands at any temperatures and decay dynamics are measured on a nanosecond scale, excluding STE contribution.

In summary, we performed a detailed study of the photoexcitation dynamics in a highlyemissive 2D perovskite compound (en) ${ }_{4} \mathrm{~Pb}_{2} \mathrm{Br}_{9} .3 \mathrm{Br}$ (MAen20) over a range of temperatures and 
excitation powers. We found that, while the PL kinetics themselves are substantially temperaturedependent, the PLQY remains remarkably nearly $T$-independent over a broad range of temperatures. Only at sufficiently high temperatures ( $T>280-290 \mathrm{~K})$, the PLQY experiences an appreciable decrease. We argued that our observations can be accommodated within a simple and physically satisfying picture of the coexisting emissive excitons (“small-size" tightly-bound states) and non-emissive geminate charge pairs ("large-size" loosely-bound states) appearing already at early relaxation times. Their $T$-dependent interconversion and dynamics explain the salient features of PL and TA kinetics as well as the onset of prevalent thermal dissociation at higher temperatures and could have important implications for the device development. Non-emissive geminate pairs could be dissociated by electric fields at donor-acceptor interfaces or by trace impurities or through edge states, ${ }^{43}$ improving the efficiency of the photovoltaic devices. Population of the emissive excitons and their binding strength, on the other hand, may be controlled via the choice of dielectric parameters, layer width and external conditions (temperature, substrate) to maximize the efficiency in light-emission devices. Hence, the results will be relevant to the rational design of high-efficiency optoelectronic devices based on the solution-processable 2D layered perovskite materials.

ASSOCIATED CONTENT

Supporting Information. Experimental methods. This material is available free of charge via the Internet at http://pubs.acs.org.

\section{AUTHOR INFORMATION}

Corresponding Author

* Email: anton.malko@utdallas.edu 


\section{ACKNOWLEDGMENT}

The work was supported by the U.S. Department of Energy, Office of Basic Energy Sciences, Division of Materials Sciences and Engineering under Award No. DE-SC0010697.

\footnotetext{
${ }^{1}$ Sutherland, B. R.; Sargent, E. H.; Perovskite photonic sources. Nat. Photonics. 2016, 10, 295302.

${ }^{2}$ Xing, G. C.; Mathews, N.; Lim, S. S.; Yantara, N.; Liu, X. F.; Sabba, D.; Gratzel, M.; Mhaisalkar, S.; Sum, T. C. Low-Temperature Solution-Processed Wavelength-Tunable Perovskites for Lasing. Nat. Mater. 2014, 13, 476-480.

${ }^{3}$ Eaton, S. W.; Lai, M.; Gibson, N. A.; Wong, A. B.; Dou, L.; Ma, J.; Wang, L.-W.; Leone, S. R.; Yang, P. Lasing in robust cesium lead halide perovskite nanowires. Proc. Natl. Acad. Sci. U.S.A 2016, 113, 1993-1998.

${ }^{4}$ Cao, Y.; Wang, N. N.; Tian, H.; Guo, J. S.; Wei, Y. Q.; Chen, H.; Miao, Y. F.; Zou, W.; Pan, K.; He, Y. R.; Cao, H.; Ke, Y.; Xu, M. M.; Wang, Y.; Yang, M.; Du, K.; Fu, Z. W.; Kong, D. C.; Dai, D. X.; Jin, Y. Z., et al. Perovskite Light-Emitting Diodes Based on Spontaneously Formed Submicrometre-Scale Structures. Nature 2018, 562, 249-253.

${ }^{5}$ Lu, M.; Zhang, Y.; Wang, S.; Guo, J.; Yu, W. W.; Rogach, A. L. Metal Halide Perovskite Light-Emitting Devices: Promising Technology for Next-Generation Displays. Adv. Funct. Mater. 2019, 1902008.
}

${ }^{6}$ Zhang, X. T.; Wang, C. C.; Zhang, Y.; Zhang, X. Y.; Wang, S. X.; Lu, M.; Cui, H. N.; Kershaw, S. V.; Yu, W. W.; Rogach, A. L. Bright Orange Electroluminescence from Lead-Free TwoDimensional Perovskites. ACS Energy Lett. 2019, 4, 242-248.

${ }^{7}$ Han, S.; Wang, P.; Zhang, J.; Liu, X.; Sun, Z.; Huang, X.; Li, L.; Ji, C.; Zhang, W.; Teng, B.; Hu W.; Hong, M.; Luo, J. Exploring a Polar Two-dimensional Multi-layered Hybrid Perovskite of $\left(\mathrm{C}_{5} \mathrm{H}_{11} \mathrm{NH}_{3}\right)_{2}\left(\mathrm{CH}_{3} \mathrm{NH}_{3}\right) \mathrm{Pb}_{2} \mathrm{I}_{7}$ for Ultrafast-Responding Photodetection. Laser Photonics Rev. 2018, 12, 1800060 .

${ }^{8}$ Zhang, Y. H.; Sun, R. J.; Qi, X. Y.; Fu, K. F.; Chen, Q. S.; Ding, Y. C.; Xu, L. J.; Liu, L. M.; Han, Y.; Malko, A. V.; Liu, X. G.; Yang, H. H.; Bakr, O. M.; Liu, H.; Mohammed, O. F. Metal Halide Perovskite Nanosheet for X-Ray High-Resolution Scintillation Imaging Screens. ACS Nano 2019, 13, 2520-2525.

${ }^{9}$ Stoumpos, C. C.; Cao, D. H.; Clark, D. J.; Young, J.; Rondinelli, J. M.; Jang, J. I.; Hupp, J. T.; Kanatzidis, M. G. Ruddlesden-Popper Hybrid Lead Iodide Perovskite 2D Homologous Semiconductors. Chem. Mater. 2016, 28, 2852-2867.

${ }^{10}$ Cheng, B.; Li, T.-Y.; Maity, P.; Wei, P.-C.; Nordlund, D.; Ho, K.-T.; Lien, D.-H.; Lin, C.-H.; Liang, R.-Z.; Miao, X.; Ajia, I. A.; Yin, J.; Sokaras, D.; Javey, A.; Roqan, I. S.; Mohammed, O. F.; He, J.-H. Extremely Reduced Dielectric Confinement in Two-Dimensional Hybrid Perovskites with Large Polar Organics. Commun. Phys. 2018, 1, 80. 
11 Yin, J.; Maity, P.; Naphade, R.; Cheng, B.; He, J.-H.; Bakr, O. M.; Brédas, J.-L.; Mohammed, O. F. Tuning Hot Carrier Cooling Dynamics by Dielectric Confinement in Two-Dimensional Hybrid Perovskite Crystals. ACS Nano 2019, 13 (11), 12621-12629.

12 Wu, X.; Trinh, M. T.; Niesner, D.; Zhu, H.; Norman, Z.; Owen, J. S.; Yaffe, O.; Kudisch, B. J.; Zhu, X.-Y. Trap States in Lead Iodide Perovskites, J. Am. Chem. Soc. 2015, 137, 2089-2096.

13 Gan, L.; Li, J.; Fang, Z.; He, H.; Ye, Z. Effects of Organic Cation Length on Exciton Recombination in Two-Dimensional Layered Lead Iodide Hybrid Perovskite Crystals, J. Phys. Chem. Lett. 2017, 8, 5177-5183.

14 Delport, G.; Chehade, G.; Lédée, F.; Diab, H.; Milesi-Brault, C.; Trippe-Allard, G.; Even, J.; Lauret, J.-S.; Deleporte, E.; Garrot, D. Exciton-Exciton Annihilation in Two-Dimensional Halide Perovskites at Room Temperature. J. Phys. Chem. Lett. 2019, 10, 5153-5159.

${ }^{15}$ Liu, J.; Leng, J.; Wu, K.; Zhang, J.; Jin, S. Observation of Internal Photoinduced Electron and Hole Separation in Hybrid Two-Dimentional Perovskite Films, J. Am. Chem. Soc. 2017, 139, 1432-1435.

${ }^{16}$ Li, M.; Gao, Q.; Liu, P.; Liao, Q.; Zhang. H.; Yao, J.; Hu, W.; Wu, Y.; Fu, H. Amplified Spontaneous Emission Based on 2D Ruddlesden-Popper Perovskites. Adv. Funct. Mater. 2018, $28,1707006$.

${ }^{17}$ Venkatesan, N. R.; Labram, J. G.; Chabinyc, M. L.; Charge-Carrier Dynamics and Crystalline Texture of Layered Ruddlesden-Popper Hybrid Lead Iodide Perovskite Thin Films. ACS Energy Lett. 2018, 3, 380-386.

${ }^{18} \mathrm{Hu}$, T.; Smith, M. D.; Dohner, E. R.; Sher, M.-J.; Wu, X.; Trinh, M. T.; Fisher, A.; Corbett, J.; Zhu, X.-Y.; Karunadasa, H. I.; Lindenberg, A. M. Mechanism for Broadband White-Light Emission from Two-Dimensional (110) Hybrid Perovskites. J. Phys. Chem. Lett. 2016, 7 , 2258-2263.

${ }^{19}$ Yin, J.; Li, H.; Cortecchia, D.; Soci, C.; Brédas, J.-L. Excitonic and Polaronic Properties of 2D Hybrid Organic-Inorganic Perovskites. ACS Energy Lett. 2017, 2, 417-423.

20 Neutzner,S.; Thouin, F.; Cortecchia, D.; Petrozza, A.; Silva, C.; Kandada, A. R. S. Excitonpolaron spectral structures in two-dimensional hybrid lead-halide perovskites, Phys. Rev. Mater. 2018, 2, 064605.

${ }^{21}$ Thouin, F.; Chávez, D. A. V.; Quarti, C.; Cortecchia, D.; Bargigia, I.; Beljonne, D.; Petrozza, A.; Silva, C.; Kandada, A. R. S. Phonon coherences reveal the polaronic character of excitons in two-dimensional lead-halide perovskites, Nat. Mater. 2019, 18, 349-356

${ }^{22}$ Miyata, K.; Meggiolaro, D.; Trinh, M. T.; Joshi, P. P.; Mosconi, E.; Jones, S. C.; Angelis, F. D.; Zhu, X.-Y. Large polarons in lead halide perovskites, Sci. Adv. 2017, 3, e1701217.

${ }^{23}$ Zheng, F.; Wang, L.-W. Large polaron formation and its effect on electron transport in hybrid perovskites, Energy Environ. Sci. 2019, 12, 1219-1230.

${ }^{24}$ Noolandi, J. Stochastic Theory of Electron-Hole Transport and Recombination in Amorphous Materials. In: Freeman, G. R. (ed). Kinetics of Nonhomogeneous Processes. Wiley, New York, 1987, p.465. 
${ }^{25}$ Pope, M.; Swenberg, C. E. Electronic Processes in Organic Crystals and Polymers. Oxford University Press, New York, 1999.

${ }^{26}$ Clarke, T. M.; Durrant, J. R. Charge Photogeneration in Organic Solar Cells. Chem. Rev. 2010, $110,6736-6767$.

${ }^{27}$ Dursun, I.; Zheng, Y.; Guo, T.; de Bastiani, M.; Turedi, B.; Sinatra, L.; Haque, Md. A.; Sun, B.; Zhumekenov, A. A.; Saidaminov, M.; de Arquer, G.; Pelayo, F.; Sargent, E.; Wu, T.; Gartstein, Y. N.; Bakr, O. M.; Mohammed O. F.; Malko, A. V. Efficient photon recycling and radiation trapping in cesium lead halide perovskite waveguides. ACS Energy Lett. 2018, 3, 1492-1498.

28 Lemmerer, A.; Billing, D. G. Lead Halide Inorganic-Organic Hybrids Incorporating Diammonium Cations. CrystEngComm. 2012, 14, 1954-1966

${ }^{29}$ Yin, J.; Maity, P.; Xu, L.; El-Zohry, A. M.; Li,H.; Bakr, O. M.; Brédas, J-L.; Mohammed, O. F. Layer-Dependent Rashba Band Splitting in 2D Hybrid Perovskites. Chem. Mater. 2018, 30, $8538-8545$.

${ }^{30}$ Lee, K. J.; Turedi, B.; Sinatra, L.; Zhumekenov, A. A.; Maity, P.; Dursun, I.; Naphade, R.; Merdad, N.; Alsalloum, A.; Oh, S.; Wehbe, N.; Hedhili, M. N.; Kang, C. H.; Subedi, R. C.; Cho, N.; Kim, J. S. Ooi, B. S.; Mohammed, O. F.; Bakr, O. M. Perovskite-Based Artificial Multiple Quantum Wells. Nano Lett. 2019, 19, 3535-3542.

${ }^{31}$ Guo, Z.; Wu, X.; Zhu, T.; Zhu, X.; Huang, L. Electron-Phonon Scattering in Atomically Thin 2D Perovskites. ACS Nano 2016, 10, 9992-9998.

32 Iwata, Y.; Banal, R. G.; Ichikawa, S.; Funato, M. Kawakami, Y. Emission Mechanisms in Alrich AlGaN/AlN Quantum Wells Assessed by Excitation Power Dependent Photoluminescence Spectroscopy. J. Appl. Phys. 2015, 17, 075701.

${ }^{33}$ Leng, K.; Abdelwahab, I.; Verzhbitskiy, I.; Telychko, M.; Chu, L.; Fu, W.; Chi, X.; Guo, N.; Chen, Z.; Zhang, C.; Xu, Q. H.; Lu, L.; Chhowalla, M.; Eda, G.; Loh, K. P. Molecularly Thin TwoDimensional Hybrid Perovskites with Tunable Optoelectronic Properties Due to Reversible Surface Relaxation. Nat. Mater. 2018, 17, 908-914.

${ }^{34}$ Anand, B.; Sampat, S.; Danilov, E.; Peng, W.; Rupich, S. M.; Gartstein, Y. N.; Chabal, Y. J.; Malko, A. V. Broadband transient absorption study of photoexcitations in lead halide perovskites: towards a multi-band picture. Phys. Rev. B: Rapid Comm. 2016, 93, 161205(R).

${ }^{35}$ Munson, K. T.; Kennehan, E. R.; Doucette, G. S.; Asbury, J. B. Dynamic Disorder Dominates Delocalization, Transport, and Recombination in Halide Perovskites. Chem 2018, 4, 2826-2843.

${ }^{36}$ Chen, X.; Lu, H.; Li, Z.; Zhai, Y.; Ndione, P. F.; Berry, J. J.; Zhu, K.; Yang, Y.; Beard, M. C. Impact of Layer Thickness on the Charge Carrier and Spin Coherence Lifetime in Two Dimensional Layered Perovskite Single Crystals, ACS Energy Lett. 2018, 3, 2273-2279.

${ }^{37}$ Malko, A.; Mikhailovsky, A.; Hollingsworth, J. A.; Petruska, M.; Klimov, V. I. Interplay between optical gain and photoinduced absorption in CdSe nanocrystals. J. Phys. Chem. B 2004, 108,5250 .

${ }^{38} \mathrm{Wu}$, X.; Trinh, M. T.; Zhu, X.-Y. Excitonic Many-Body Interactions in Two-Dimensional Lead Iodide Perovskite Quantum Wells. J. Phys. Chem. C 2015, 119, 14714-14721. 
39 Lantry, G.; Jemli, K.; Wei, Y.; Leymarie, J.; Even, J.; Lauret, J.-S.; Deleporte, E. RoomTemperature Optical Tunability and Inhomogeneous Broadening in 2D-Layered OrganicInorganic Perovskite Pseudobinary Alloys. J. Phys. Chem. Lett. 2014, 5, 3958-3963.

${ }^{40}$ Yaffe, O.; Guo, Y.; Tan, L. Z.; Egger, D. A.; Hull, T.; Stoumpos, C. C.; Zheng, F. Heinz, T. F.; Kronik, L. Kanatzidis, M. G.; Owen, J. S.; Rappe, A. M.; Pimenta, M. A.; Brus, L. E. Local Polar Fluctuations in Lead Halide Perovskite Crystals. Phys. Rev. Lett. 2017, 128, 136001.

${ }^{41}$ Ni, L.; Huynh, U.; Cheminal, A.; Thomas, T. H.; Shivanna, R.; Hinrichsen, T. F.; Ahmad, S.; Sadhanala, A.; Rao, A. Real-Time Observation of Exciton-Phonon Coupling Dynamics in SelfAssembled Hybrid Perovskite Quantum Wells. ACS Nano 2017, 11, 10834-10843.

${ }^{42}$ Mauck, C. M.; Tisdale, W. A. Excitons in 2D Organic-Inorganic Halide Perovskites. Trends in Chemistry 2019, 4, 380-393.

43 Blancon, J.-C.; Tsai, H.; Nie, W.; Stoumpos, C. C.; Pedesseau, L; Katan, C.; Kepenekian, M.; Soe, C. M. M.; Appavoo, K.; Sfeir, M. Y.; Tretiak, S.; Ajayan, P. M.; Kanatzidis, M. G.; Even, J.; Crochet, J. J.; Mohite, A. D. Extremely Efficient Internal Exciton Dissociation Through Edge States in Layered 2D Perovskites. Science 2017, 355, 1288-1292. 